http://jmscr.igmpublication.org/home/ ISSN (e)-2347-176x ISSN (p) 2455-0450 crossref DOI: https://dx.doi.org/10.18535/jmscr/v8i3.91

Journal Of Medical Science And Clinical Research

\title{
Comparison of Clinical Profile of Pleural Effusion with Special References to Various Biochemical Parameters in Patients Admitted to Government Tertiary Care Center
}

\author{
Authors \\ Dr S.V.Birajdar ${ }^{1}$, Dr Dinesh Mohan Chaudhari ${ }^{2}$, Dr S.S.Chavan ${ }^{3}$, Dr Vishal Lede ${ }^{4}$ \\ ${ }^{1}$ Prof and Head of Department, ${ }^{2}$ Resident, ${ }^{3}$ Aso Prof., ${ }^{4}$ Lect \\ Department of General Medicine, S.R.T.R.GMCH Ambajogai (Maharashtra)
}

\section{Introduction}

A pleural effusion is collection of fluid abnormally present in the pleural space, usually resulting from excess fluid production and/or decreased lymphatic absorption presenting with cardiopulmonary symptoms. A systemic approach to the investigations is needed because of the extensive differential diagnosis

A pleural effusion is collection of fluid abnormally present in the pleural space, usually resulting from excess fluid production and/or decreased lymphatic absorption. ${ }^{1}$ It is the most common manifestation of pleural disease, and its etiologies range in spectrum from cardiopulmonary disorders and/or systemic inflammatory conditions to malignancy. It is one of the major causes of pulmonary morbidity and mortality

Mechanisms by which the rate of fluid formation exceeds the rate of fluid absorption include increased pulmonary capillary pressure or permeability of the endothelial barrier, decreased intra pleural pressure or plasma oncotic pressure, obstructed lymphatic flow, diaphragmatic defects, and thoracic duct rupture. ${ }^{2}$ Pleural fluid aspiration followed by application of the time-honored Light's criteria remains a remarkably useful step in the diagnostic algorithm of pleural effusions, although an expanding panel of diagnostic tools in the past decade has slowly transformed our approach to pleural diseases, the increasing complexity of which has motivated the development of multidisciplinary pleural subspecialty programs at many institutions in recent years. ${ }^{3,4}$

Worldwide, exudative effusions are usually due to empyema, malignancy, tuberculosis, pulmonary embolism, and connective tissue diseases. ${ }^{5}$

In our setup, the common causes of exudative pleural effusions are tuberculosis, parapneumonic effusion, and malignancy. In developing nations, infections- especially tuberculosis and parapneumonic effusions, are more prevalent. ${ }^{6}$

Diagnosis of effusion requires a proper clinical, radiological, histological evaluation along with cytological study. Fluid cytology is a cost effective, rapid and highly efficient tool for the evaluation of body information provided by body fluid analysis serve several functions as it assists the clinician in formulating in order of priority of 
differential diagnosis and also allows one to follow the result of therapy. ${ }^{5}$ In this study an attempt has be determine the diagnostic yield of fluid cytology to reach the etiological diagnosis of pleural and peritoneal effusion.

In this study, we will attempt to summarize what are the clinical and biochemical parameters of pleural effusion. Even with this high risk of getting pleural effusion in so many different diseases, specific researches are lacking in studying these clinical spectrum in our country. This study gives the brief overview of clinical presentations and laboratory findings of all those patients admitted to Medicine department of a tertiary care hospital.

\section{Aims and Objective}

1. To study the clinical and biochemical profile of various types of pleural effusions.

2. Comparison of various biochemical parameters used to differentiate pleural effusion in transudate and exudate

3. To find out various risk factors of pleural effusion.

\section{Materials and Methods}

Study Centre: Medicine department of tertiary care hospital in Maharashtra

Study Duration: Duration of study is 24 months, From Nov 2017 to Oct 2019

Study population: Patients admitted with pleural effusion in the department of medicine were included in the study.

Study Design: Cross-sectional Observational study.

\section{Inclusion Criteria}

1. Any case of Pleural effusion

2. Age : Above 13 years

\section{Exclusion Criteria}

1. Age $<13$ years

2. Already diagnosed case of pleural effusion

3. Patients not willing to participate in the study.

\section{Methodology}

All patients admitted with pleural effusion in the department of medicine were included in the study. All these patients were subjected to detailed clinical history regarding their presenting complaints, other symptoms like breathlessness, chest pain, cough with sputum production, fever etc. were enquired.

Detailed clinical examination was carried out and with all routine investigations.

All the patients were subjected to Diagnostic Pleurocentesis. Under aseptic precautions about $10 \mathrm{ml}$ of fluid was aspirated and subjected to pleural fluid analysis -

Biochemical, Pathological analyses were done. Pleural fluid Sugar, Protein, LDH, was measured for all patients. Pleural fluid ADA levels were estimated for exudative effusions in affordable patients. Pleural fluid cell count and cytology were done in all patients. Pleural fluid gram staining, AFB staining, Culture and sensitivity tests were carried out in all patients. Informed consent was obtained for all the invasive procedures prior to it.

Other investigations like Echocardiography, Ultra sonogram abdomen were done in relevant cases only. All the patients were subjected to HIV screening by ELISA technique. All the patients were studied in an every possible way and an appropriate etiological diagnosis was made out in a systematic way.

\section{Results}

Age and sex wise distribution of pleural effusion Most of the patients were between the age group of 21-30 and 41-50 and they constituted the $38.88 \%$ and pleural effusion was more common in males (61. In our study mean age of TB Pleural Effusion was 45.5. 


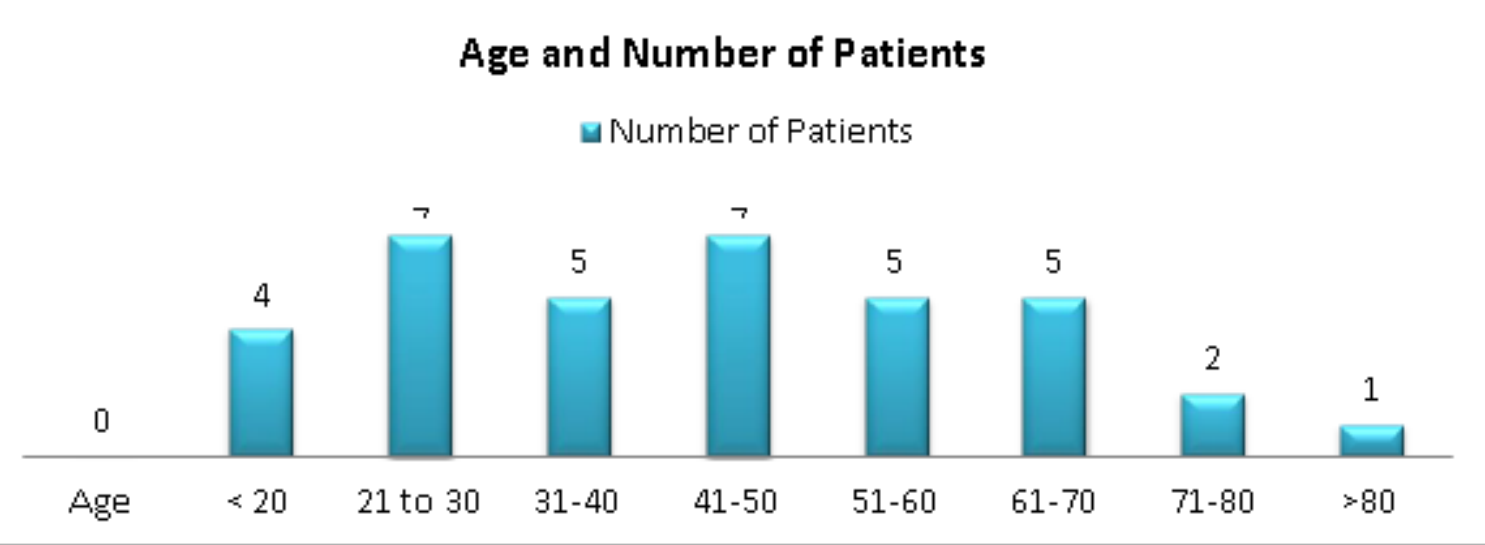

Fig. 1 Age and distribution of patients of pleural effusion Maximum cases showed male preponderance, that was $22(61 \%)$ and females $14(39 \%)$.

\section{Gender}

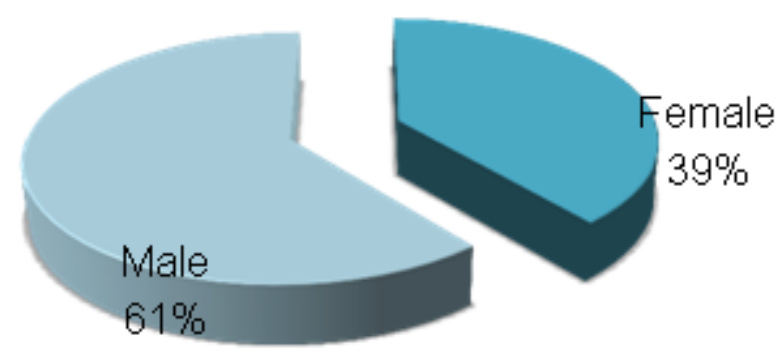

Fig 2 Gender and distribution of patients of pleural effusion

Table.1 Occupation and prevalence of tubercular pleural effusion according to the occupation.

\begin{tabular}{|l|c|c|c|c|}
\hline Occupation & $\begin{array}{c}\text { Number of } \\
\text { patients }\end{array}$ & Percentage & bercular pleural effusion & $\begin{array}{c}\text { centage of tubercular pleural } \\
\text { effusion }\end{array}$ \\
\hline Driver & 4 & $11.11 \%$ & 2 & $50 \%$ \\
\hline Farmer & 9 & $25 \%$ & 7 & $78 \%$ \\
\hline House Wife & 6 & $16.67 \%$ & 4 & $67 \%$ \\
\hline Laborer & 8 & $22.22 \%$ & 7 & $88 \%$ \\
\hline Shopkeeper & 3 & $8.33 \%$ & 2 & $67 \%$ \\
\hline Student & 6 & $16.67 \%$ & 4 & $67 \%$ \\
\hline
\end{tabular}

In this study prevalence of pleural effusion was high among farmers and that was $25 \%$ of study population. The prevalence of tubercular effusion was high among laborer that was $88 \%$.

Table 2 Etiology of pleural effusion

\begin{tabular}{|l|c|}
\hline Diagnosis & Number of cases (Percentage \%) \\
\hline Tubercular pleural effusion & $25(69.44 \%)$ \\
\hline Malignant Pleural effusion & $3(8.33 \%)$ \\
\hline IHD DM CCF & $2(5.55 \%)$ \\
\hline Other (Synpneumonic Effusion, Empyema, SLE) & $6(16.66 \%)$ \\
\hline
\end{tabular}

Table 2 suggests that out of 36 cases maximum numbers of cases of pleural effusion were tubercular, $25(69.44 \%)$ cases. Out of $66.66 \%$ patients of tubercular effusion, 5 (13.88\%) patients were diabetic, $3(8.33 \%)$ patient were hypertensive and $2(5.55 \%)$ patients had ischemic 
heart disease.

As shown in table 3, the most common symptom was Cough and fever in $91.66 \%$, followed by chest pain (88.88\%) and breathlessness $77.77 \%$. Other symptoms were expectoration in $41.66 \%$ of patients, hemoptysis $13.88 \%$, loss of weight in $44.44 \%$ patients, swelling of feet in $16.66 \%$ and clot formation was seen in $75 \%$ of patients.

Right sided pleural effusion was more common that of left side.

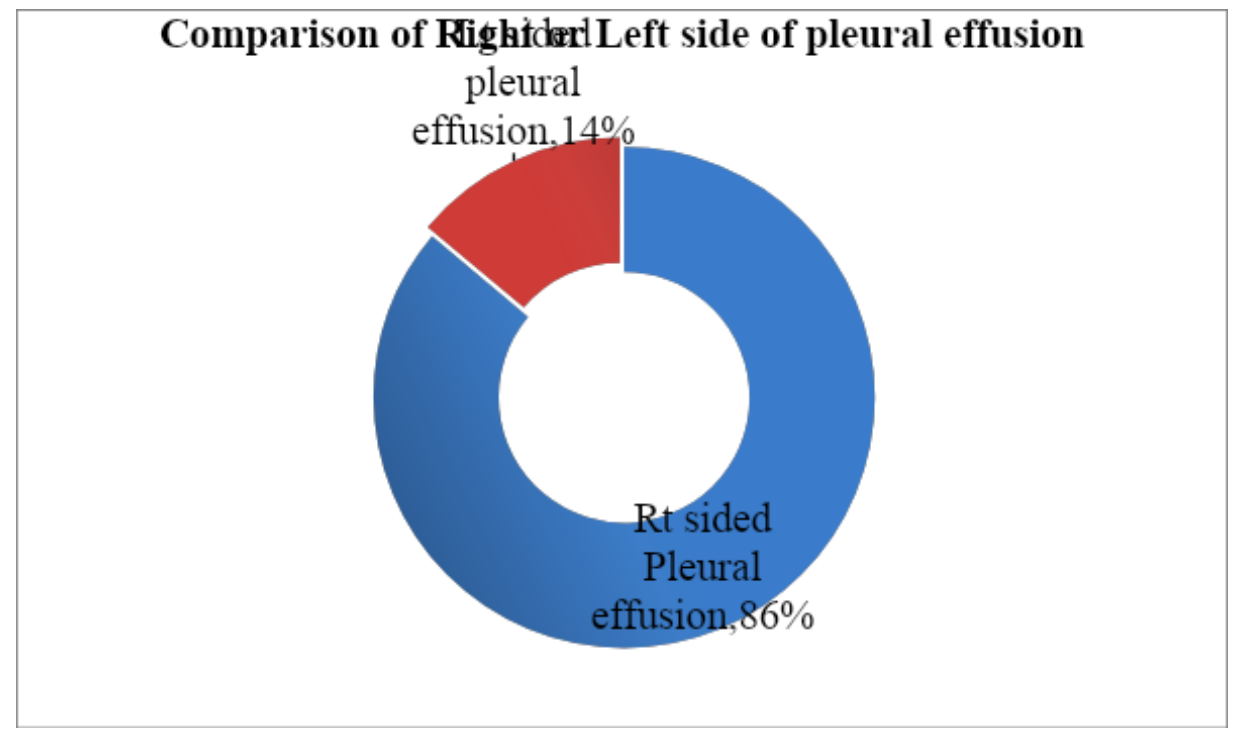

Fig. 3 Comparison of Right or Left side of pleural effusion

Table.4 Appearance of pleural fluid

\begin{tabular}{|l|c|c|}
\hline Appearance of pleural Fluid No of cases: & Number & Percentage \\
\hline Yellowish Turbid & 24 & $66.67 \%$ \\
\hline Reddish turbid & 7 & $19.44 \%$ \\
\hline Straw colored & 2 & $5.56 \%$ \\
\hline Turbid & 2 & $5.56 \%$ \\
\hline Turbid with puss like & 1 & $2.78 \%$ \\
\hline
\end{tabular}

Table 5 Pleural fluid ADA levels

\begin{tabular}{|l|c|c|c|}
\hline ADA Level & $<\mathbf{4 0}$ & $\mathbf{4 1 - 6 0}$ & $>\mathbf{6 0}$ \\
\hline Tubercular & - & $5(13.88 \%)$ & $20(55.55 \%)$ \\
\hline Congestive cardiac failure & $2(5.55 \%)$ & - & - \\
\hline Malignant & $1(2.77 \%)$ & $2(5.55 \%)$ & - \\
\hline SLE with pleural effusion & $1(2.77 \%)$ & - & - \\
\hline Synpneumonic Effusion & $1(2.77 \%)$ & - & - \\
\hline other & $2(5.55 \%)$ & - & - \\
\hline
\end{tabular}

Table.6 Pleural fluid protein

\begin{tabular}{|l|c|c|}
\hline Pleural fluid protein & $\mathbf{4 - 6 ~ g m \%}$ & $\mathbf{6 g m} \%$ \\
\hline Tubercular & $17(47.22 \%)$ & $8(22.22 \%)$ \\
\hline Congestive cardiac failure & $2(5.55 \%)$ & - \\
\hline Malignant & $2(5.55 \%)$ & $1(2.77 \%)$ \\
\hline SLE with left sided pleural effusion & $1(2.77 \%)$ & - \\
\hline Synpneumonic Effusion & - & $1(2.77 \%)$ \\
\hline Other & $2(5.55 \%)$ & - \\
\hline
\end{tabular}

Pleural fluid CBNAAT was done in all these cases, CBNAAT was positive in 5 cases, confirming the presence of $\mathrm{M}$. tuberculosis in specimen of these patients. 
Table. Total leucocyte count (TLC) of pleural Fluid

\begin{tabular}{|l|c|c|}
\hline TLC & Below 5000 & above 5000 \\
\hline Number of patients & 6 & 30 \\
\hline Percentage & $16.67 \%$ & $83.33 \%$ \\
\hline
\end{tabular}

\section{Pleural fluid Glucose}

A low pleural fluid glucose $(<60 \mathrm{mg} / \mathrm{dL})$ was mainly caused by TB and rheumatoid pleuritis, complicated parapneumonic effusion, malignancy, and caused rarely hemothorax, paragonimiasis, Churg-Strauss syndrome, and occasionally, lupus pleuritis.

Pleural fluid levels were below $60 \mathrm{mg} / \mathrm{dL}$ in 5 cases $(13.88 \%)$ and in 31 cases it was above $60 \mathrm{mg} / \mathrm{dL}(86.12 \%)$

\section{Discussion}

In the present study pleural effusions were more common in 21-30 and 41-50 years of age group which is comparable with the study of Burgers and Pujan P. ${ }^{7,8}$ In our study mean age of TB Pleural Effusion was 45.5. In the present study group male to female ratio was $1.56: 1$, which is comparable with the studies of Maldhure (2.13:1), and Hirsch (2.53:1). ${ }^{9,} 10$

In this study prevalence of pleural effusion was high among farmers that are $25 \%$ of study population with pleural effusion was farmers, and laborer, among this prevalence of tubercular effusion was high among laborer that is $88 \%$.

Pleural effusion is caused by pulmonary or nonpulmonary diseases. Although the etiologic spectrum is wide, most effusions occur due to tuberculosis, malignancy, heart failure. In our study common etiology was tuberculosis as it well studied that in India tuberculosis prevalence is high. While in various other studies incidence of tubercular pleural effusion was low, this can be explained by the fact that tuberculosis is more prevalent in India. On cytology most common cases of pleural effusion were of chronic effusion $(86.1 \%)$ followed by inflammatory and malignancy. This result is similar to the study of Anita B et al 4 in which out of 31 cases of pleural effusion 24 cases $(77.4 \%)$ were of chronic effusion. Lymphocytes were predominantly seen in patients with chronic effusion. Fluid cytology is a useful, rapid and a highly sensitive method for the diagnosis of effusions. Cytological examination of fluids is a simple and minimally invasive step in the diagnostic work up of patients. It has high diagnostic sensitivity and helps the clinician in formulating the correct algorithm for treatment purpose. Integration of fluid cytology with clinical and laboratory findings further increases the diagnostic yield. In developing country like India where health facilities are inadequate and cost of investigations and management in often unaffordable, fluid analysis and cytology should continue to be the first line of investigation to screen out the malignant and suspicious for malignancy effusion cases.

Most common presentation was right sided pleural effusion (86\%) which is comparable with the study of Ambethiya P (right side pleural effusion76\%) and Gosh B K (right side pleural effusion$80.2 \%$ ), This was also supported by studies done by Laim et al, and Marel et al. ${ }^{11,} 12,13$ In Tuberculous Right lung is more commonly involved, hence pleural effusion more common to occurs than left. In the present study incidence of exudative effusion was $91 \%$ which was comparable with the study of Amethiya P (94\%). In various other studies the incidence of exudative effusion was low which can be explained by the fact that tuberculosis is more prevalent in India having exudative etiology.

Incidence of malignant pleural effusion was $8.3 \%$ which was comparable with the Indian studies of Gosh B K (10.2\%) ${ }^{11}$

Pleural fluid ADA was the most important factor for diagnosing TB Pleural Effusion. In $83.33 \%$ patient of TB Pleural Effusion pleural fluid ADA was more than 40. In 6 patients pleural fluid ADA was in the range of below 40 . The higher the level the more likely of TB. It was supported by studies of Liang et al. ${ }^{14}$ In association with lymphocytic pleural effusion ADA has $95 \%$ sensitivity and $89 \%$ specificity in TB Pleural Effusion. ${ }^{15}$

A low pleural fluid glucose $(<60 \mathrm{mg} / \mathrm{dL})$ is mainly 
caused by TB and rheumatoid pleuritis, complicated parapneumonic effusion, malignancy, and caused rarely hemothorax, paragonimiasis, Churg-Strauss syndrome, and occasionally, lupus pleuritis. Total leucocyte count was above 5000 in 30 cases $(83.33 \%)$ and was below 5000 in 6 (16.67\%) cases. Among pleural effusion patients Lymphocytic predominant cases were 29 $(80.56 \%)$

Pleural fluid CBNAAT was done in all these cases, CBNAAT was positive in 2 cases

The most common symptoms were Cough and fever in $91.66 \%$, followed by chest pain $(88.88 \%)$ and breathlessness $77.77 \%$. Similar results were found in studies of Kausal et al and Godwin et al. ${ }^{16,17}$ Pleural fluid CBNAAT was done in all these cases, CBNAAT was positive in 5 cases, confirming the presence of

M. tuberculosis in specimen of these patients. Conventional smear microscopy, often used as a first line of diagnostic tool for diagnosis of pulmonary tuberculosis being a simple, economical, and easy-to-do test, could not provide diagnosis in any subjects in present study. Apart from being an operator dependent test, it needs over 10,000 bacilli per $\mathrm{ml}$ to give a positive result. In present study, cartridge based nucleic acid amplification test (CBNAAT) detected M. tuberculosis in pleural fluid samples of 5 cases out of 36 cases. Additionally, mycobacterial susceptibility to rifampicin was ascertained in all these five patients. The Xpert MTB/RIF test exhibits high sensitivity and specificity for detecting pulmonary TB disease. CBNAAT is having unmatched significance in TB endemic areas like India where there is high prevalence of MDR-TB. Pleural effusions in patients with congestive heart failure are typically bilateral. In our study, CCF accounted for only $5.55 \%$ of cases. Synpneumonic Effusion, Empyema, SLE are less frequent accounting for $16.66 \%$ of cases in our study.

\section{Conclusion}

In conclusion of this study, tuberculous pleural effusion was most common cause followed by malignant pleural effusion and IHD with CCF. Farmers and laborers as occupation were common occupation in our study population. This is similar to what is being seen in many studies conducted across developing countries. Hence, we conclude that intensive antitubercular measures may go a long way in bring down the number of patients presenting with pleural effusion. This study was intended to provide a systematic approach to investigate the patient of Pleural Effusion so as early necessary treatment can be initiated without delay.

\section{Reference}

1. Diaz-Guzman E, Dweik RA. Diagnosis and management of pleural effusions: a practical approach. Compr Ther. 2007 Winter. 33(4):237-46.

2. Akulian J, Feller-Kopman D. The past, current and future of diagnosis and management of pleural disease. $\mathbf{J}$ Thorac Dis 2015;7:S329-38.

3. Bhatnagar $\mathrm{R}$, Maskell $\mathrm{N}$ : Developing a 'pleural team' to run a reactive pleural service. Clin Med (Lond). 2013;13(5): 4526.

4. Diaz-Guzman E, Dweik RA. Diagnosis and management of pleural effusions: A practical approach. Compr Ther 2007;33: 237-46.

5. Sahn SA. The differential diagnosis of pleural effusions. West J Med 1982;137: 99108

6. Evidence-based clinical practice guidelines. Chest. 2013 May. 143 (5 Suppl): e455S97S.

7. Burgess, LJ, Maritz, FJ, Le Roux, I. Combined use of pleural adenosine deaminase with lymphocyte/neutrophil ratio: increased specifi city for the diagnosis of tuberculous pleuritis. Chest. 1996;109,414-9.

8. Parikh P, Odhwani J, Ganagajalia C. Study of 100 cases of pleural effusion with 
reference to diagnostic approach. Int J Adv Med 2016;3:328-331.

9. Maldhure BR, Bedarkar SP, Kulkarnl HR, Paplnwar SP. Pleural biopsy and adenosine deaminase in pleural fluid for the diagnosis of tubercular pleural effusion. Ind $\mathrm{J}$ Tub. 1994;41:161-5

10. Hirsch A, Ruffie P, Nebut M. Pleural effusion: laboratory tests in 300 cases. Thorax. 1979;34(1):106-12.

11. Ghosh B. K, Bandyopadhyay U. A Study of Etiology and Clinical Profile of Patient with Pleural Effusion in a Teaching Hospital." IOSR Journal of Dental and Medical Sciences (IOSR-JDMS), vol. 18, no. 9, 2019, pp 19-23.

12. Marel M, Arustova M, Stasny B et al. Incidence of pleural effusion in a welldefined region: epidemiologic study in central Bohemia. Chest 1993;104:14861489.

13. Laim CK, Wong CM. Causes of pleural exudates in a region with a high incidence of tuberculosis. Respirology. 2000;5:33-38.

14. Liang QL, Shi HZ, Wang $K$ et al. Diagnostic accuracy of adenosine deaminase in tuberculous pleurisy: A metaanalysis. Respir Med, 2008;102:744-754.

15. Diaacon AH, Van de wal BW, Wyser C, Smedema JP, Bezuidentront J, Bolliger CT, Walzi G: Diagnostic tools in tuberculous pleurisy : a direct comparative study. Eur Respir J 2003;22: 589-591.

16. Godwin C M, Ajuonuma B. C, Ofondu E. O, Aguwa E.1 N. Pleural effusion: Aetiology, clinical presentation and mortality outcome in a tertiary health institution in Eastern Nigeria - A five year retrospective study. J AIDS Clin Res 2015;6:2.

17. Kaushal MB, Krupal PM. Pleural effusion: A two years prospective study in western India. Sch J App Med Sci. 2015;3(8A): 2790-793. 